\title{
Very Early Robot-Assisted Mobilization of Intensive Care Patients - A Scoping Review
}

\author{
Amrei C. KLAMT ${ }^{\mathrm{a}, 1}$, Lena SCHMIDBAUER ${ }^{\mathrm{a}}$, Angelika WARMBEIN ${ }^{\mathrm{b}}$, Ivanka \\ RATHGEBER $^{\mathrm{b}}$, Uli FISCHER ${ }^{\mathrm{b}}$ and Inge EBERL ${ }^{\mathrm{a}}$ \\ ${ }^{a}$ Catholic University of Eichstätt-Ingolstadt, Kapuzinergasse 2, 85072 Eichstätt, \\ Germany \\ ${ }^{\mathrm{b}}$ Hospital of the Ludwig-Maximilians-University (LMU) Munich, Marchioninistr. 15, \\ 81377 Munich, Germany
}

\begin{abstract}
This scoping review gives an overview of current research activities in the field of very early mobilization with robotic devices of intensive care patients. It presents the effect of very early, robot-assisted mobilization on intensive care patients based on their outcomes.
\end{abstract}

Keywords: intensive care patients, very early mobilization, rehabilitation, robotics.

\section{Introduction}

The mobilization of intensive care patients requires high personnel costs [1] and presents, to some extent, a safety risk to all those involved. Studies show that early mobilization can have a positive influence on the healing process and the rehabilitation of critically ill patients [2]. Robotic systems should help to carry out early mobilization using fewer human resources and therefore make it feasible to perform during the everyday work of intensive wards. This scoping review gives an overview of previous research on the early robot-assisted mobilization of intensive care patients. It addressed the following research questions: how is early mobilization carried out on intensive wards using robotic systems? What effect does early mobilization carried out using robotic systems have on patient outcomes?

\section{Methods}

In order to answer these research questions, a systematic literature search was undertaken in accordance with the PRISMA extension for scoping reviews [3]. A search was conducted from May to July 2020 using defined search strings in the following databases: MEDLINE (via PubMed and Web of Science), CINAHL, Cochrane Library, Embase, IEEE Xplore, Scopus and WTI. An extended search took place using ResearchGate and GoogleScholar. Articles were included in the search that contained rehabilitation

\footnotetext{
${ }^{1}$ Corresponding Author, Catholic University of Eichstätt-Ingolstadt, Department of nursing science, Kapuzinergasse 2, 85072 Eichstätt, Germany; E-mail: Amrei.Klamt@ku.de.
} 
robotics, (early) mobilization robotics and transfer robotics, with the aim of application on intensive wards.

\section{Results}

A total of 996 articles were found. After removing the duplicates, 869 articles remained. A double-blind screening of titles and abstracts reduced this number further to 41 articles. Following a full-text screening, 14 articles remained, which were then assessed on their methodical composition with regard to quality and evidence using the Critical Appraisal Tools [4] of the Joanna Briggs Institute and were then selected for the study. The 14 articles encompass four texts and opinions, a case report, two case-control studies, a quasi-experimental intervention study, a cross-sectional study and five RCTs.

\section{Discussion}

The early robot-assisted mobilization of intensive care patients has previously mainly taken place using electronic bed-mounted exercise bicycles, sometimes combined with muscle stimulation. In addition, electronic treadmills or tilt tables are used, which, however, always require the patient to be transferred to the therapeutic equipment. This inevitably carries a safety risk for those involved. The introduction of these early mobilization systems has a positive effect on patient outcomes, demonstrated by a decrease in the incidence of the onset of delirium, the stability of respiration and improved bodily function [5].

\section{Conclusions}

The findings show that further research into the use of robotic systems in early mobilization is required. In particular, the systems currently in use have not yet led to optimal savings in human resources. It is also clear that robotic systems can usefully supplement but not replace conventional early mobilization.

\section{References}

[1] Rai S, Anthony L, Needham DM, Georgousopoulou EN, Sudheer B, Brown R, Mitchell I, van Haren F. Barriers to rehabilitation after critical illness: a survey of multidisciplinary healthcare professionals caring for ICU survivors in an acute care hospital. Australian Critical Care. 2020;33(3):264-71.

[2] Waldauf P, Jiroutková K, Krajčová A, Puthucheary Z, Duška F. Effects of Rehabilitation Interventions on Clinical Outcomes in Critically Ill Patients: Systematic Review and Meta-Analysis of Randomized Controlled Trials. CRIT CARE MED. 2020;48(7):1055-65.

[3] Tricco AC, Lillie E, Zarin W, O'Brien KK, et al. PRISMA Extension for Scoping Reviews (PRISMAScR): Checklist and Explanation. Ann Intern Med. 2018 Oct 2;169(7):467-473.

[4] The Joanna Briggs Institute (JBI). Critical Appraisal Tools, Available at https://joannabriggs.org/criticalappraisal-tools, Accessed Dec 16, 2020.

[5] Parry SM, Berney S, Koopman R, Bryant A, El-Ansary D, Puthucheary Z, Hart N, Warrillow S, Denehy L. Early rehabilitation in critical care (eRiCC): functional electrical stimulation with cycling protocol for a randomised controlled trial. BMJ Open. 2012;2(5). 